\title{
Cómo Mide la Inteligencia la Escala Terman. Modelo Psicométrico General. Normas Revisadas y Actualización de Resultados
}

\section{How the Terman Scale Measures Intelligence. General Psychometric Model. Revised Rules and Updated Results}

\author{
Juan José Ballesteros Domingo \\ EOEP Centro-Arganzuela Madrid, España
}

\begin{abstract}
Resumen. Dos son las causas por las que la escala Terman da lugar a medidas de CI significativa y progresivamente más altas que las obtenidas por las actuales escalas factoriales: la irregular variabilidad del CI entre diferentes niveles de edad y capacidad (variabilidad atípica) y el desfase de los resultados con el paso del tiempo. A partir de la tesis de que los rendimientos de los sujetos tienden a conformar distribuciones de puntuaciones típicas derivadas y de la determinación de la variabilidad natural propia de las escalas Terman, se define un Modelo Psicométrico General que se relaciona con los modelos de las escalas factoriales. A continuación, se propone un procedimiento para corregir la variabilidad atípica y determinar Normas Alternativas Proporcionales. Finalmente, se describe el peculiar patrón de desfase del CI de las escalas de edad (la dinámica del CI) y se ofrece una estimación, a partir de las normas proporcionales, de los valores actuales del CI.

Palabras clave: Escala Terman, medida de la inteligencia, revisión de normas, actualización de valores de CI.
\end{abstract}

\begin{abstract}
There are two reasons why the Terman Scale produces IQ values significantly and progressively higher than those obtained with the currently used factor scales: the irregular variability of IQ among different age and ability levels (atypical variability) and the results becoming outdated as time goes on. Based on the thesis that the performance of the individuals tends to form distributions of typical derivative scorings and from the determination of the natural variability characteristic of the Terman scales, we define a General Psychometric Model which is related to the models of the factor scales. Next, we propose a procedure to correct the atypical variability and to determine Proportional Alternative Rules. Lastly, the peculiar pattern of the outdated IQ of the age scales (the IQ dynamics) is described, and an estimation of the actual IQ values is provided using the proportional rules.
\end{abstract}

Keywords: Terman Scale, measurement of intelligence, rules revision, update of IQ values.

El principio de normalidad ha determinado el tratamiento estadístico de las escalas de medida de la inteligencia. Es uno de los pocos aspectos sobre los que no existe discusión ni alternativa. En las escalas

La correspondencia sobre este artículo debe enviarse al e-mail: juanjoseballes@yahoo.es factoriales, hoy hegemónicas, los rendimientos directos de los sujetos en las pruebas (raw scores) se normalizan, buscando dispersiones homogéneas entre las diferentes edades. Los parámetros básicos, media y desviación típica, se eligen arbitrariamente en función de la comodidad de utilización para obte- 
ner un CI de desviación. En la escala Terman, máximo exponente de las escalas de edad tipo Binet, los rendimientos brutos de los sujetos en el total de la escala generan directamente distribuciones normales de media y dispersión crecientes con la edad cronológica (EC).

En este artículo sostengo la tesis de que, si los criterios de paso están suficientemente ajustados y las muestras son suficientemente representativas, la escala Terman tiende a generar distribuciones de puntuaciones típicas derivadas para los diferentes niveles de EC. Aunque los primeros rendimientos no son medibles, este tipo de escalas tienen origen común en $E M_{0}=0$ y la relación $\overline{E M} / S_{E M}=6$ para todas las EC. Como consecuencia, la escala de CI en la que todas se transforman, tiene asimismo origen en $C I_{0}=0$. De la estructura de la escala se deduce la dispersión a la que tienden los rendimientos (EM) y los valores del CI, que califico como dispersión natural. Su determinación permite generalizar el modelo psicométrico de la escala Terman y proponer normas alternativas proporcionales, que suprimen la variabilidad atípica de los valores del CI en la Segunda y en las dos versiones de la Tercera Edición.

\section{La Estimación del CI}

\section{La Naturaleza de las Pruebas que conforman la Escala Terman}

Las pruebas de la escala tratan de verificar si los sujetos alcanzan el mínimo establecido para superarlas (criterio de paso), no de buscar el máximo rendimiento en cada tarea. Están organizadas en niveles de seis para cada edad cronológica de referencia (EC). Los criterios de paso de las pruebas que forman cada uno de estos niveles son superados por una mayoría suficiente de los sujetos de la correspondiente EC (típicamente por encima del 60 por ciento si la escala está idealmente ajustada). La superación de cada prueba suma al rendimiento final dos meses de edad mental (EM) (un solo mes en las edades inferiores, con niveles cada seis meses). Las pruebas de las escalas de edad son de suficiencia $\mathrm{o}$ de mínimos, no de rendimiento.

Cada sujeto realiza un reducido número de las 152 pruebas que componen la escala completa, empezando (si no hay otros datos sobre su capacidad) por aquellas seis que suelen superar los de capacidad media de su misma EC. Según su capacidad avanzan o retroceden sus propios niveles en la escala. Cuanto más separadas están las edades de los sujetos menos pruebas comparten, llegando a ser totalmente diferentes. Incluso en dos casos de similar edad, si se prevé que los resultados van a dar rendimientos opuestos y efectivamente los dan, las pruebas que realicen serán diferentes. Por tanto, cada sujeto al ser valorado por la escala realiza $s u$ propia y particular escala. En contraste, en las pruebas factoriales todos los sujetos realizan todas las pruebas, alcanzando en ellas la máxima puntuación que le permiten sus capacidades.

\section{La Dispersión Natural de los Rendimientos y sus Consecuencias}

Se conoce como CI de ejecución el obtenido a través de la escala Terman (y de las escalas de edad en general), en contraposición al CI de desviación propio de las escalas factoriales, que siempre necesita normas para su obtención. Esta distinción entre ambos CI se refiere a los diferentes procedimientos seguidos para obtener la valoración de la inteligencia. Pero con frecuencia produce la confusión de pensar que las escalas de edad miden directamente valores de CI y su dispersión $\left(S_{C I}\right)$, cuando lo que realmente recogen son los rendimientos de los sujetos (EM) y la dispersión de estos rendimientos $\left(S_{E M}\right)$. La fuente de la variabilidad atípica está en los rendimientos, donde debe ser corregida para evitar que se traslade al CI.

Se puede considerar la escala como la unión de seis series longitudinales y paralelas $(p)$, teniendo cada una de ellas una prueba por nivel de EC. Los rendimientos de muestras representativas para cada nivel de EC conforman, en cada serie, distri- 
buciones de puntuaciones típicas derivadas de forma:

$e m_{p, i}=\overline{e m}_{p} \times\left(z_{p, i}+1\right)$

En este tipo de distribuciones la media coincide con la desviación típica:

$\overline{e m}_{p}=S_{e m p}$

Cada sujeto tiene un rendimiento potencialmente diferente en cada serie, siendo su rendimiento en el total de la escala $\left(E M_{i}\right)$ la suma de los rendimientos parciales en las seis series $\left(e m_{p i}\right)$ :

$E M_{i}=\sum_{\mathrm{p}=1}^{6} e m_{p, i}$

Y el rendimiento medio $(\overline{E M})$ en cada nivel de EC:

$$
E M=\sum_{\mathrm{p}=1}^{6} \overline{e m}_{p}
$$

Como en el ajuste ideal las seis pruebas de cada nivel de EC son equivalentes, los rendimientos medios de cada serie $\left(\overline{e m}_{p}\right)$ son iguales, y por tanto también lo son sus desviaciones típicas $\left(S_{e m p}\right)$. La desviación típica de la escala $\left(S_{E M}\right)$ resulta ser:

$S_{E M}=\sqrt{\left(\sum_{\mathrm{p}=1}^{6} S_{e m_{\mathrm{p}}}^{2}\right) / 6}$

$S_{E M}=S_{e m_{p}}$

La conclusión es que, en cada nivel de EC de la escala idealmente ajustada, el rendimiento medio es seis veces mayor que la desviación típica:

$\overline{E M}=6 \times S_{E M}$

La dispersión natural a la que tiende la escala (que a partir de aquí identifico como $S^{\prime}$ ) depende idealmente de su estructura, es decir del número de pruebas por nivel de EC ( $p)$, no de las poblaciones o épocas sobre las que se determinen normas. Al identificarse la escala del CI con la del grupo ideal de $\overline{E M}=100$, la dispersión natural del CI en la escala Terman resulta ser:
$\overline{C I}=6 \times S_{C I}$

$S_{C I}^{\prime}=16.66$

En otros términos el cociente de variación $(\mathrm{CV})$ para todos los niveles de edad es 1/6. Las edades, las poblaciones y las épocas difieren en sus rendimientos medios $(\overline{E M})$, pero tienen en común la dispersión natural. Determinar esta dispersión hace posible el ajuste proporcional de los rendimientos y la construcción sin distorsiones de las normas del CI.

\section{Modelo Psicométrico Ideal de la Escala Terman}

La escala Terman supone el máximo desarrollo de las escalas de edad tipo Binet. En su forma ideal debe cumplir las condiciones psicométricas descritas por sus autores (Terman, L. M. y Merrill, M. A. 1943):

El primer punto que tiene que satisfacer la tipificación de una escala de inteligencia del tipo de la de BINET es que la edad mental media de un grupo de sujetos sin seleccionar de cualquier edad cronológica concuerde íntimamente con la edad cronológica media, o, expresado en otra forma, que sujetos sin seleccionar de cualquier edad logren un C.I. medio de 100, aproximadamente. (p. 38).

El empleo de los valores de C.I. se basa en la presunción de que la variabilidad en términos del C.I. permanece aproximadamente constante en las diversas edades, o, dicho de otra forma, que la variabilidad en términos de E.M. es directamente proporcional a la E.C. Sólo en tanto esta presunción sea cierta puede tener el C.I. un significado firme y estable, pues, de lo contrario, un C.I. determinado en una cierta edad podría ser equivalente a otro C.I. mucho más alto o más bajo de otra edad. (p. 43).

En resumen, en la escala ideal debe cumplirse, para todos los niveles de $E C$ :

1. distribución normal de los rendimientos $(E M)$.

2. $\overline{E M}=E C, \mathrm{y}$

3. $S_{C I}$ constante. 
El valor preciso de $S_{C I}$ no se determina, aunque la estimación realizada para la Segunda Edición se acerca a los 17 puntos (Terman, L. M. y Merrill, M. A. 1943, p. 45). Bajo los supuestos anteriores puede aplicarse la fórmula clásica para la obtención del CI:

$$
C I_{i}=\left(E M_{i} / E C\right) \times 100
$$

que con la sustitución de $B=100 / E C$, queda expresada como:

$$
C I_{i}=B \times E M_{i}
$$

La constante ideal $B$, propia para cada EC, indica la estricta proporcionalidad entre los rendimientos de EM y sus valores de CI.

Se ajusta la dificultad de las pruebas que conforman la escala, aplicándolas a muestras experimentales, hasta que en todas las muestras representativas de edad (formadas por sujetos diferentes a las anteriores) los resultados sean normales con $\overline{E M}$ coincidentes con la EC y con dispersiones homogéneas. Para aumentar objetivamente el grado de dificultad de la escala de edad pueden elevarse los criterios de paso de las pruebas, resituarlas en niveles inferiores y/o eventualmente sustituirlas por otras. No es necesario determinar normas de CI para la escala (que se obtiene por el cociente clásico) ya que están implícitas en el ajuste progresivo de la dificultad de las pruebas.

La Segunda Edición de la escala Terman (publicada en 1937), con formas paralelas L y M, fue la última basada en el modelo psicométrico ideal y elaborada exclusivamente por el procedimiento experimental. En el propio manual se describen con detalle los pasos seguidos y las numerosas dificultades encontradas, entre las que destaca la de controlar la diferente dispersión de las puntuaciones en algunas de las EC.

Los autores explican la variabilidad atípica del CI (significativamente alta a los 2 y medio y a los 12 años y significativamente baja a los 6 años) por las deficiencias en la selección de las muestras de tipificación (Terman, L. M. y Merrill, M. A. 1943, p. 43). Por su parte, McNemar (1942, p. 85) atribuye la variabilidad atípica, al menos parcialmente, a los porcentajes de paso por edad de las pruebas utilizadas y a un posible artefacto de la propia escala. En la misma obra (ps. 172-174) publica tablas de ajuste del CI, por grupos de edad, que tratan de corregir esta variabilidad desde los rendimientos medios. En el Apéndice se propone una corrección de las normas de la Segunda Edición, con el mismo procedimiento que, para la Tercera Edición, se determina en los apartados siguientes. Conviene destacar que los resultados son opuestos a los propuestos en su día por Quinn McNemar.

\section{Modelo Psicométrico General de la Escala Terman}

La sustitución en la ecuación clásica de EC (medida en meses de tiempo) por $\overline{E M}$ (medida en meses de rendimiento) y la determinación de la dispersión natural de los rendimientos permiten generalizar el modelo psicométrico. El CI se identifica ahora con el rendimiento de un grupo de edad ideal con $\overline{E M}=100$.

El modelo psicométrico general que aquí se propone queda determinado por los siguientes supuestos para todos los niveles de EC:

1. distribución normal de los rendimientos $(E M)$.

2. $\overline{E M} / S_{E M}=6$.

Si las dispersiones se ajustan a sus valores ideales, el $C I$ se obtiene igualmente de la relación $S_{C I}$ / $S_{E M}$ :

$C I_{i}=\left(S_{C I} / S_{E M}\right) \times E M_{i}$

La constante $B=100 / E C$ del modelo ideal se sustituye por la constante $B^{\prime}=100 / \overline{E M}$, manteniendo la proporcionalidad entre rendimientos y valores de $C I$.

\section{Jerarquía de Modelos Psicométricos}

Las escalas factoriales de medida de la inteligencia se basan en un modelo psicométrico que permi- 
te combinar los resultados de diferentes pruebas, que miden diferentes capacidades intelectuales, en un CI total. El supuesto fundamental de las escalas factoriales es la normalidad de los diferentes rendimientos de las pruebas utilizadas. Los rendimientos de cada prueba $\left(X_{i}\right)$, medidos en su propia escala, se transforman por niveles de edad en puntuaciones típicas con media y desviación típica arbitrarias (por ejemplo, $\bar{Y}=10$ y $S_{Y}=3$ ), mediante la ecuación de transformación de puntuaciones típicas:

$Y_{i}=10+\left(\frac{\mathrm{X}_{\mathrm{i}}-\overline{\mathrm{X}}}{S x}\right) \times 3$

Los distintos rendimientos en las pruebas, que responden a diferentes capacidades, se pueden reunir así en valores típicos intermedios (subescalas) y valor típico o escalar total $P T_{i}$ ) del que se obtiene el CI general del sujeto con parámetros arbitrarios, habitualmente $\overline{C I}=100$ y $S_{C I}=15$ :

$C I_{i}=100+\left(\frac{P T_{i}-\overline{\mathrm{PT}}}{S_{P T}}\right) \times 15$

El modelo psicométrico general de la escala Terman resulta ser un caso particular del modelo factorial, en el que, debido a la estructura ya descrita de las pruebas, el rendimiento total de cada sujeto $\left(E M_{i}\right)$ es directamente un valor perteneciente a la escala típica derivada de su nivel de edad. Por tanto, utiliza la misma ecuación de transformación de puntuaciones típicas:

$C I_{i}=100+\left(\frac{E M_{i}-\overline{E M}}{S_{E M}}\right) \times S_{C I}^{,}$

que puede expresarse en forma operativa:

$C I_{i}=100-\frac{\overline{E M}}{S_{E M}} \times S_{C I}{ }_{C I}+\frac{S_{C I}^{\prime}}{S_{E M}} \times E M_{i}$

Siendo las constantes: $a=100\left(E M / S_{E M}\right) \times S_{C I}^{\prime}$ y $b=\left(S_{C I}{ } / S_{E M}\right)$, la relación en tre EM y CI para cada EC se puede asimismo expresar como función lineal:

$C I_{i}=a+b \times\left(E M_{i}\right)$
Como $S_{C I}=16.66$ y $C V=1 / 6$, para todos los niveles de edad, la constante es nula y la ecuación 16 se simplifica:

$C I_{i}=\frac{S_{C I}^{\prime}}{S_{E M}} \times E M_{i}$

Resultando ser la misma ecuación 12 de la fórmula del CI de ejecución. El CI de ejecución, cuando los rendimientos están ajustados, se calcula con la fórmula del CI de desviación. El Modelo Ideal es, a su vez, un caso restringido y particular del Modelo General en el que $\overline{E M}=E C$ para todos los niveles de edad.

\section{La Variabilidad Atípica de las normas de la Tercera Edición}

En 1960 aparece la Tercera Edición de la Escala Terman, con una sola forma combinada L-M que es la que se mantiene invariable hasta hoy, para la que S.R. Pinneau opta por un procedimiento de normalización propio de las escalas factoriales, entonces en pleno desarrollo. Una década después (1972), R. L. Thorndike elabora las últimas normas.

El procedimiento de normalización seguido en 1972 es descrito ya con detalle por W. H. Angoff (1971, pp. 588 y ss.). En esencia, se obtiene la distribución común de CI, con $\overline{C I}=100$ y $S_{C I}=16$, empleando la ecuación 15 de transformación de puntuaciones típicas.

La dispersión del CI está determinada por la constante $b$, mientras que la combinación de ambas constantes $a$ y $b$ ajustan el rendimiento medio $(\overline{E M})$ al . Como en general $a \neq 0$, las normas, manteniendo la relación lineal entre rendimientos y valores correspondientes de CI, pierden la proporcionalidad exigida por los modelos psicométricos de la escala, enunciados anteriormente.

El resultado son valores de $C I$ que cumpliendo la condición de normalidad para todos los niveles de edad, trasladan la variabilidad atípica de los rendimientos de EM a la escala de CI pero no la suprimen. Las normas presentan desviaciones en diferen- 
te grado para cada EC, que aumentan a medida que se distancian de la media. Este resultado se indica en el propio manual:

Hay que señalar también que para cualquier nivel de ejecución o E.M. conseguida, las desviaciones no son las mismas para diversos niveles de habilidad. En general, las desviaciones son mayores para rendimientos medios y superiores de los más jóvenes o para aquellos cuya EM, está por encima de su E.C. y menores para los jóvenes de rendimiento por debajo de la media de habilidad, llegando a una mínima desviación, si la hay, en los niveles más bajos de habilidad. Así, pues, las deviaciones representadas en las tablas de C.I. de 1972 no son sólo función del grupo de sujetos de la misma E.C., sino de su habilidad dentro del grupo. (Terman, L. M. y Merrill, M. A. 1972, p. 415).

Los sujetos reciben finalmente un valor mayor o menor de CI no sólo en función de sus capacidades sino también en función de la edad a la que son evaluados. Si, bajo el supuesto habitual, mantienen constantes sus capacidades irán obteniendo valores de CI significativamente diferentes en posibles aplicaciones sucesivas de la escala. En el Gráfico 1 se representan los cambios de CI según el valor obtenido en la primera ejecución de la escala y para las edades iniciales de 3, 5, 6 y 8 años. Efectivamente, las mayores variaciones se producen para los sujetos más jóvenes y de mayor rendimiento.

\section{Determinación de Normas Alternativas Proporcionales}

Para hacer operativo el modelo general debe determinarse la constante $B$ ' para cada nivel de EC. Se parte de las normas de 1972 estableciendo un procedimiento general para su corrección. Previamente, se modifican las constantes $a$ y $b$ de las ecuaciones originales, sustituyendo el valor arbitrario de la dispersión por su valor natural antes indicado $S_{\mathrm{CI}}{ }_{\mathrm{CI}}$ $=16.66$ (Tabla 1 ). Las constantes $a$ y $b$ empleadas en los cálculos serán a partir de aquí las obtenidas con la dispersión natural del CI.

Gráfico 1. Escala Terman (1972). Evolución del CI para un mismo sujeto según la edad y el CI obtenido inicialmente

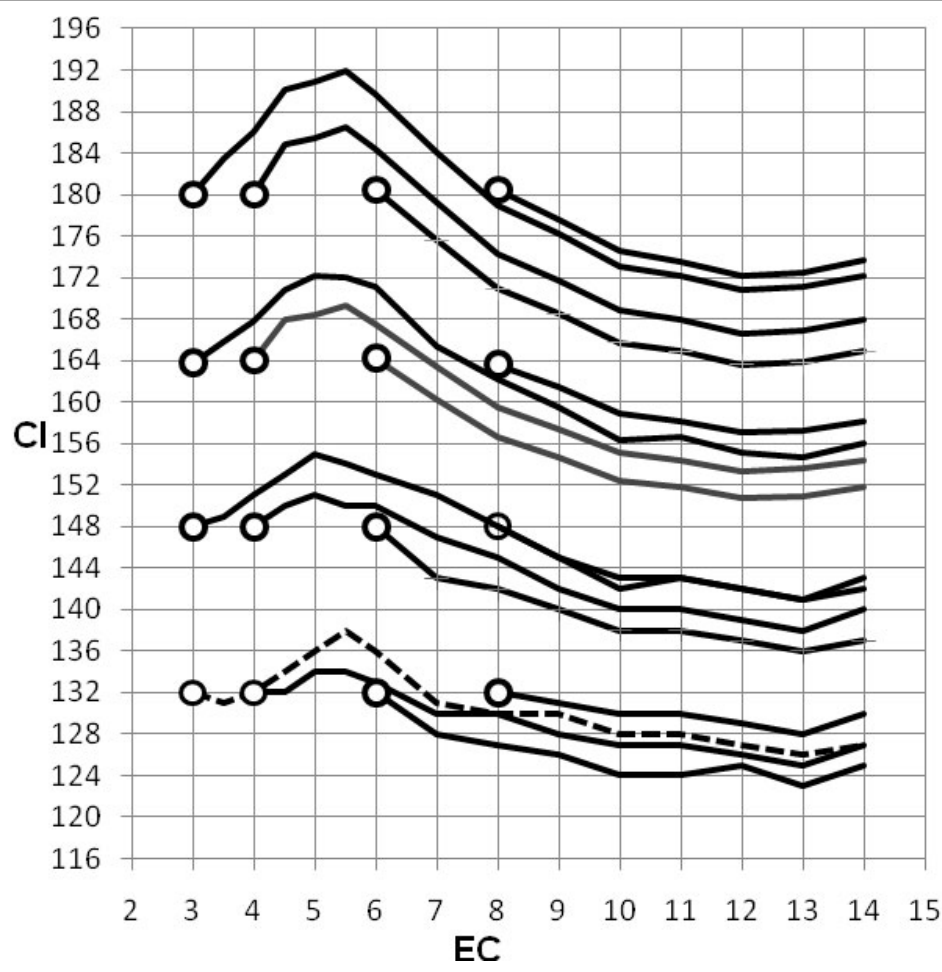


Tabla 1. Escala Terman: Constantes y parámetros de las Normas entre las EC de 3 y 14 años

\begin{tabular}{|c|c|c|c|c|c|c|c|}
\hline \multirow[b]{2}{*}{$\mathrm{EC}$} & \multirow[b]{2}{*}{ (meses) } & \multicolumn{2}{|c|}{$S_{C I}-16$} & \multicolumn{2}{|c|}{ (Tercera Edición-1972) } & \multicolumn{2}{|c|}{$S_{C I}^{\prime}-16.66$} \\
\hline & & $a$ & $b$ & $\overline{E M}$ & $S_{E M}$ & $a$ & $b$ \\
\hline 3 años & 36 & -2.185 & 2.4408 & 41.87 & 6.56 & -6.450 & 2.5424 \\
\hline $3 \mathrm{a} 6 \mathrm{~m}$ & 42 & -5.469 & 2.2040 & 47.85 & 7.26 & -9.851 & 2.2957 \\
\hline 4 años & 48 & -8.750 & 2.0187 & 53.87 & 7.93 & -13.274 & 2.1027 \\
\hline $4 \mathrm{a} 6 \mathrm{~m}$ & 54 & -13.879 & 1.9007 & 59.91 & 8.42 & -18.611 & 1.9798 \\
\hline 5 años & 60 & -14.785 & 1.7498 & 65.60 & 9.14 & -19.565 & 1.8226 \\
\hline $5 \mathrm{a} 6 \mathrm{~m}$ & 66 & -16.180 & 1.6370 & 70.97 & 9.77 & -21.014 & 1.7051 \\
\hline 6 años & 72 & -13.194 & 1.4785 & 76.56 & 10.82 & -17.906 & 1.5400 \\
\hline 7 años & 84 & -6.323 & 1.2050 & 88.23 & 13.28 & -10.743 & 1.2552 \\
\hline 8 años & 96 & 0.211 & 0.9905 & 100.75 & 16.15 & -3.947 & 1.0317 \\
\hline 9 años & 108 & 3.701 & 0.8518 & 113.05 & 18.78 & -0.304 & 0.8873 \\
\hline 10 años & 120 & 7.590 & 0.7292 & 126.73 & 21.94 & 3.742 & 0.7596 \\
\hline 11 años & 132 & 8.757 & 0.6617 & 137.89 & 24.18 & 4.960 & 0.6892 \\
\hline 12 años & 144 & 10.538 & 0.5956 & 150.20 & 26.86 & 6.817 & 0.6204 \\
\hline 13 años & 156 & 10.211 & 0.5542 & 162.02 & 28.87 & 6.471 & 0.5773 \\
\hline 14 años & 168 & 8.722 & 0.5291 & 172.51 & 30.24 & 4.926 & 0.5511 \\
\hline
\end{tabular}

\section{Cálculo de la constante B'}

Se toma como referencia para la corrección proporcional de normas para cada EC el rendimiento $E M_{X}$ al que corresponde el CI de 100 antes de aplicar el ajuste con la constante $a$, Este rendimiento $E M_{X}$ es el único con valor de CI no distorsionado, ya que la corrección factorial sobre 100 de $a$ unidades es equivalente a la corrección proporcional de $a$ (en porcentaje). El rendimiento $E M_{X}$ es siempre equivalente a seis unidades típicas:

$$
\begin{aligned}
& E M_{X}=6 \times S_{E M} \\
& E M_{X}=\frac{100}{S_{C I}^{\prime}} \times S_{E M}
\end{aligned}
$$

Su correspondiente $C I_{X}$ se puede calcular mediante la ecuación 16 :

$$
C I_{X}=100-\frac{\overline{E M}}{S_{E M}} \times S_{C I}^{{ }_{C I}}+\frac{S_{C I}^{\prime}}{S_{E M}} \times\left[\frac{100}{S_{C I}^{\prime}} \times S_{E M}\right]
$$

Alternativamente, $C I_{X}=100+a$. La relación entre $C I_{X}$ y $E M_{X}$ determina la constante $B$ ' buscada, que permite el cálculo del CI sin distorsión $\left(C I^{\prime}\right)$ :

$B^{\prime}=\frac{C I_{X}}{E M_{\mathrm{X}}}$

$C I_{i}^{\prime}=B^{\prime} \times E M_{i}$
Las normas alternativas obtenidas, ajustando las escalas desde las $E M_{X}$, son estrictamente proporcionales y, redondeando los valores calculados, las $\overline{E M}$ no se modifican en muchos casos o tienen variaciones no significativas. Pero la constante $B$ ' es sensiblemente menor que la constante $b$ en las edades de los más jóvenes. Para obtener los $C I^{\prime}$ de edades intermedias basta con extrapolar proporcionalmente los valores asignados a las EC superior e inferior. Los valores $E M_{X}, C I_{X}$, las constantes $B^{\prime}$ y $b^{\prime}$ (que se define en el siguiente apartado), y los parámetros corregidos $\left(\overline{E M}\right.$, y $S_{E M}$ ) se indican en la Tabla 2.

\section{Fórmula General del CI’de Ejecución}

La constante $B$ ' obtenida transforma directamente los rendimientos $E M_{i}$ en valores de $C I_{i}^{\prime}$ proporcionales. Su cálculo en función de $\overline{E M}$ y $S_{E M}$ se deduce de la combinación de las ecuaciones 22, 21 y 20:

$$
\begin{aligned}
& B^{\prime}=\left[100-\frac{\overline{E M}}{S_{E M}} \times S_{C I}^{\prime}+\frac{100 \times S_{E M}}{S_{C I}}\right] /\left[\frac{100 \times S_{E M}}{S^{\prime}{ }_{C I}}\right] \\
& B^{\prime}=\left[2-\frac{\overline{E M}}{6 \times S_{E M}}\right] \times\left[\frac{S_{C I}}{S_{E M}}\right]
\end{aligned}
$$

La fórmula general de obtención del CI proporcional ( $\left.C I^{\prime}\right)$, expresada en función de $\left(\overline{E M}\right.$ y $S_{E M}$ es: 
Tabla 2: Escala Terman. Normas Alternativas Proporcionales a las de 1972 entre las EC de 3 y 14 años $\left(S_{C I}{ }_{C I}-16.66\right)$

\begin{tabular}{|c|c|c|c|c|c|c|c|}
\hline EC & (meses) & $E M_{X}$ & $C I_{X}$ & $b^{\prime}$ & $B^{\prime}$ & $\overline{E M}$ & $S_{E M^{\prime}}$ \\
\hline 3 años & 36 & 39.33 & 93.56 & 0.936 & 2.3787 & 42.04 & 7.01 \\
\hline 3 a $6 \mathrm{~m}$ & 42 & 43.56 & 90.15 & 0.901 & 2.0696 & 48.32 & 8.05 \\
\hline 4 años & 48 & 47.56 & 86.73 & 0.867 & 1.8236 & 54.84 & 9.14 \\
\hline 4 a $6 \mathrm{~m}$ & 54 & 50.51 & 81.39 & 0.814 & 1.6114 & 62.06 & 10.34 \\
\hline 5 años & 60 & 54.87 & 80.44 & 0.804 & 1.4660 & 68.21 & 11.37 \\
\hline 5 a $6 \mathrm{~m}$ & 66 & 58.65 & 78.99 & 0.790 & 1.3468 & 74.25 & 12.37 \\
\hline 6 años & 72 & 64.93 & 82.09 & 0.821 & 1.2643 & 79.10 & 13.18 \\
\hline 7 años & 84 & 79.67 & 89.26 & 0.893 & 1.1203 & 89.26 & 14.88 \\
\hline 8 años & 96 & 96.92 & 96.05 & 0.961 & 0.9910 & 100.91 & 16.82 \\
\hline 9 años & 108 & 112.71 & 99.70 & 0.997 & 0.8846 & 113.05 & 18.84 \\
\hline 10 años & 120 & 131.66 & 103.74 & 1.037 & 0.7880 & 126.91 & 21.15 \\
\hline 11 años & 132 & 145.09 & 104.96 & 1.050 & 0.7234 & 138.23 & 23.04 \\
\hline 12 años & 144 & 161.19 & 106.82 & 1.068 & 0.6627 & 150.90 & 25.15 \\
\hline 13 años & 156 & 173.23 & 106.47 & 1.065 & 0.6146 & 162.70 & 27.12 \\
\hline 14 años & 168 & 181.45 & 104.93 & 1.049 & 0.5783 & 172.93 & 28.82 \\
\hline
\end{tabular}

$C I_{i}^{\prime}=\left[2-\frac{\overline{E M}}{6 \times S_{E M}}\right] \times\left[\frac{S_{C I}}{S_{E M}}\right] \times E M_{i}$

Identificando como $b^{\prime}$ y $b$ respectivamente los dos factores que componen la constante $B^{\prime}$ :

$C I_{i}^{\prime}=b^{\prime} \times b \times E M_{i}$

\section{Características y efectos de la constante b'}

El segundo factor de la constante $B$ ' es la constan-

te $b$ de las normas factoriales. El primer factor $b$ ':
$b^{\prime}=\left[2-\frac{\overline{E M}}{6 \times S_{E M}}\right]$

Alternativamente se puede expresar en función de la constante $a$ :

$b^{\prime}=1+a / 100$

La expresión del CI' resulta, en función de las constantes a y b:

$$
C I_{i}^{\prime}=(1+a / 100) \times b \times E M_{i}
$$

Gráfico 2. Escala Terman. Comparación del ajuste de normas con las constantes $a$ factorial y b’ proporcional

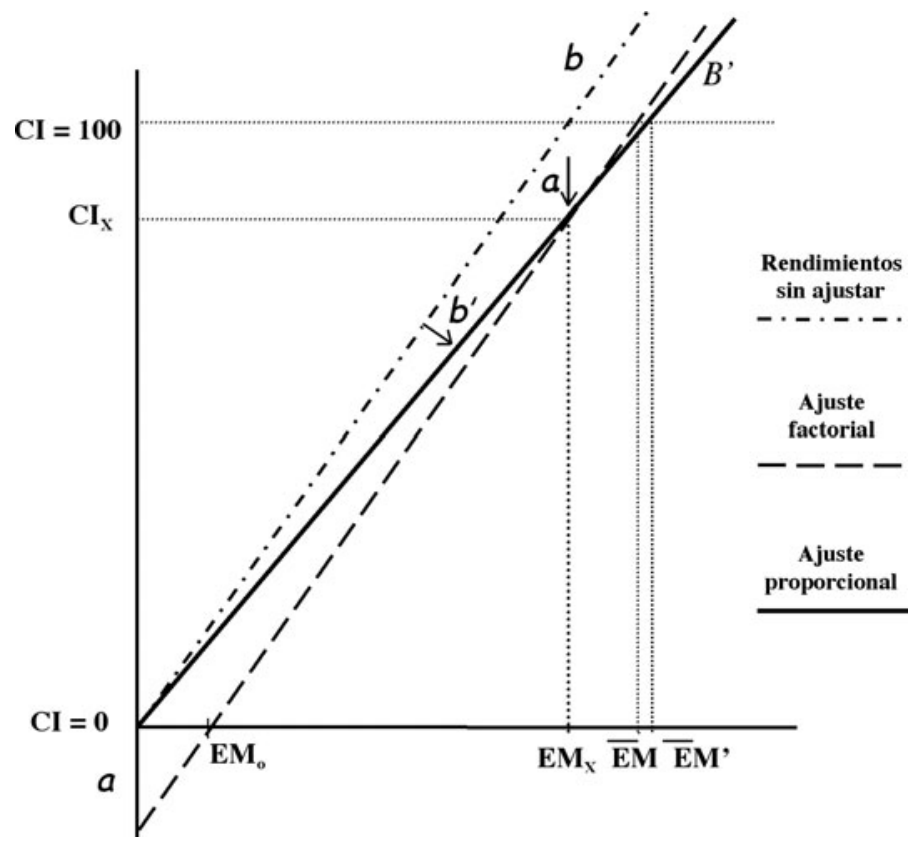


Los diferentes procedimientos de ajuste de las normas se representan en el Gráfico 2. Se ha elegido el caso en que $a<0$, ya que es el que da lugar a las mayores correcciones de los valores del $C I$, afectando sobre todo a las edades en torno a los cinco años y medio.

El exceso de valoración del $C I$ para la $\overline{E M}$ de la distribución de rendimientos es la constante $a$, que en el ajuste factorial de normas reduce todos los rendimientos por igual. Los rendimientos bajos quedan reducidos en mayor proporción que los altos, produciéndose así la distorsión de las normas. Además, la $\overline{E M}$ deja de ser la mediana de la distribución de rendimientos. La constante $b$ ', de acuerdo a la segunda condición del Modelo Psicométrico General, ajusta proporcionalmente los rendimientos. Con el aumento de la dispersión de los rendimientos (pendiente $B$ ' pendiente $b$ ) aumenta el rendimiento medio $(\overline{E M}$ ' > $\overline{E M})$. El producto de todos los valores de una distribución normal por una constante (en este caso $b$ ') no modifica su CV. La constante $b$ ' aumenta proporcionalmente $E M_{X}$ hasta su conversión en el rendimiento medio de la escala corregida $(\overline{E M}$ '). Por tanto, la nueva $\overline{E M}$ 'no procede de la $\overline{E M}$, sino del rendimiento $E M_{X}$, referencia para la determinación de las normas alternativas:

$\overline{E M}{ }^{\prime}=E M_{X} / b^{\prime}$

Conocida la constante $b^{\prime}$, se puede aplicar directamente para transformar las distribuciones normales de los rendimientos de cada nivel de $E C$ empleados en las normas originales $\left(E M_{i}\right)$ en otras, también normales, de rendimientos $\left(E M_{i}^{\prime}\right)$ y dispersión ideal $S_{E M}^{\prime}$ :

$$
\begin{aligned}
& E M_{i}^{\prime}=E M_{i} / b^{\prime} \\
& S_{E M^{\prime}}=S_{E M} / b^{\prime}
\end{aligned}
$$

Para las nuevas distribuciones EM' la ecuación de transformación de puntuaciones típicas y la clásica de obtención del CI de ejecución son equivalentes:

$$
\begin{aligned}
& C I_{i}^{\prime}=100 \frac{\overline{E M^{\prime}}}{S_{E M}} \times S_{C I}{ }_{C I}+\frac{S_{C I}^{\prime}}{S_{E M}} \times E M_{i}^{\prime} \\
& C I_{i}^{\prime}=\frac{S_{C I}^{\prime}}{S_{E M^{\prime}}} \times E M_{i}^{\prime} \\
& C I_{i}^{\prime}=\frac{100}{E M}, \times E M_{i}^{\prime}
\end{aligned}
$$

\section{La Dinámica del CI}

Cuando los rendimientos de una población en la escala mejoran de forma significativa, las normas establecidas los transforman en mayores valores de CI. Aparentemente se produce un aumento del CI. De igual manera que los rendimientos extremadamente bajos no son medibles con la escala, la asunción de la normalidad de rendimientos nos lleva a considerar que la acumulación de casos con resultados claramente por encima de las cuatro unidades típicas son un indicativo del desfase de las normas. Conviene destacar que los sujetos no aumentan directamente su CI, sino su rendimiento en las escalas que lo miden (en este caso en unidades de EM). $\mathrm{El} \mathrm{CI}$ (que carece de unidad) confronta el rendimiento individual con las normas para la edad, población y época correspondientes. Si las normas quedan anticuadas, los valores de CI obtenidos resultan mayores de lo que deberían ser. En otros términos, la inflación del CI se debe corregir con la devaluación del rendimiento (EM), ya que en caso contrario terminamos comparando el nivel de los sujetos actuales con el de sus antepasados.

Por otra parte, la mejora general de la población en la ejecución de las escalas de inteligencia no se debe al progreso uniforme en todos los niveles de rendimiento o habilidad. En términos gráficos, la curva normal de rendimiento (y por tanto la del CI) no se desplaza hacia su izquierda por traslación en bloque. El modelo psicométrico de la escala Terman asocia el aumento general del rendimiento $(\overline{E M})$, que se distribuye normalmente, con un aumento proporcional de su dispersión $\left(S_{E M}\right)$. Gráficamente, la curva normal que representa al CI permanece anclada en los niveles mínimos de ejecución y se despla- 
za hacia su izquierda por expansión aumentando simultáneamente la media y la dispersión de sus valores, manteniendo constante la relación entre ambas $(\mathrm{CV}=1 / 6)$.

El desfase de las normas de 1960 a 1972 puede aportar información relevante al debate sobre cuánto aumenta el CI con el paso del tiempo. El aumento del rendimiento se puede atribuir directamente a la mejora de la capacidad de la población, ya que la prueba se mantiene inalterada. En la Tabla 3 se ofrecen los rendimientos medios corregidos $(\overline{E M}$ ') para las normas de 1960, calculados por el procedimiento empleado anteriormente, comparándolos con los de 1972. casos en que ya no se utiliza la escala). Los valores que sufren finalmente mayor alteración son los más altos de los sujetos de menor edad (precisamente en aquellos casos en que se sigue aplicando la escala). En la Tabla 4 se indican algunos ejemplos de corrección del CI y posterior actualización, suponiendo que las aplicaciones de la escala se hicieran en la década actual.

Los resultados de los cálculos ofrecidos en la Tabla 4 deben ser interpretados como tendencias generales, sin atribuirles la precisión que no tienen. Quien considere los argumentos aquí expuestos y trate de obtener un valoración orientativa de un resultado concreto de CI deberá tomas ciertas pre-

Tabla 3. Escala Terman. Diferencias entre los rendimientos medios corregidos () de los tres a los catorce años para las normas de 1972 y de 1960 , en valores absolutos y porcentajes

\begin{tabular}{|c|c|c|c|c|c|}
\hline $\mathrm{EC}$ & (meses) & $\overline{E M} '(60)$ & $\overline{E M}^{\prime}(72)$ & Diferencias & Diferencias $(\%)$ \\
\hline 3 años & 36 & 37.54 & 42.04 & 4.52 & 12.31 \\
\hline 3 a $6 \mathrm{~m}$ & 42 & 42.92 & 48.32 & 5.62 & 13.09 \\
\hline 4 años & 48 & 48.77 & 54.84 & 6.42 & 13.16 \\
\hline 4 a $6 \mathrm{~m}$ & 54 & 54.82 & 62.06 & 7.84 & 14.30 \\
\hline 5 años & 60 & 61.38 & 68.21 & 7.53 & 12.27 \\
\hline 5 a $6 \mathrm{~m}$ & 66 & 67.94 & 74.25 & 7.14 & 10.51 \\
\hline 6 años & 72 & 73.66 & 79.10 & 6.16 & 8.36 \\
\hline 7 años & 84 & 85.89 & 89.26 & 3.83 & 4.46 \\
\hline 8 años & 96 & 98.53 & 100.91 & 2.55 & 2.59 \\
\hline 9 años & 108 & 111.12 & 113.05 & 1.95 & 1.75 \\
\hline 10 años & 120 & 123.43 & 126.91 & 3.29 & 2.67 \\
\hline 11 años & 132 & 134.80 & 138.23 & 3.16 & 2.34 \\
\hline 12 años & 144 & 146.57 & 150.90 & 3.94 & 2.69 \\
\hline 13 años & 156 & 157.85 & 162.70 & 4.44 & 2.81 \\
\hline 14 años & 168 & 166.78 & 172.93 & 5.82 & 3.49 \\
\hline Promedios: & & & & 4.95 & 7.12 \\
\hline
\end{tabular}

El aumento del rendimiento medio en una década, para las edades entre tres y catorce años, fue globalmente cercano al cinco por ciento. Se puede observar que los aumentos son significativamente superiores para los más jóvenes. Si aceptamos unos 4 puntos de mejora en el CI por década, para ajustarlos a la época actual tendremos que reducir los valores sin distorsión del CI en un $16 \%$ aproximadamente.

Sobre la variabilidad atípica original de las valoraciones del CI se va acumulando el desfase con el paso del tiempo. Antes de estimar el valor actual del CI hay que corregir su distorsión. Los valores de CI más estables resultan ser los correspondientes a los rendimientos más bajos (precisamente para los cauciones. Debido a que en el manual no se imprimieron valores más allá de las cuatro desviaciones típicas, en muchas ocasiones se ha calculado el CI con las Tablas de Conversión del Apéndice A (Terman, L. M. y Merrill, M. A. 1975, pp.391 y ss.), que pertenece a las normas de 1960 y ofrecen sistemáticamente mayores valores de CI. En otras ocasiones, se utiliza sin más la fórmula clásica, de la que se obtienen valores igualmente erróneos. Por tanto, deberá tener la seguridad de que el CI se obtuvo realmente con las normas de 1972. En caso contrario, deberá partir de la EC y la fecha de realización de la prueba utilizando realmente las normas de 1972 como base de los cálculos. 
Tabla 4. Escala Terman. Ejemplos de valores de CI obtenidos en la actual década de los 10, según las normas de 1972 y de su actualización

\begin{tabular}{|c|c|c|c|c|c|c|c|}
\hline \multirow[b]{2}{*}{$\mathrm{EC}$} & \multirow[b]{2}{*}{ EM } & \multicolumn{2}{|c|}{ Corrección del CI } & \multicolumn{3}{|c|}{ Actualización del CI' (por décadas) } & \multirow[b]{2}{*}{ Dif. } \\
\hline & & CI - 72 & CI' & $4 \%$ & Dif. & $5 \%$ & \\
\hline 36 m (3 a) & $55 \mathrm{~m}$ & 132 & 130 & 109 & -23 & 104 & -28 \\
\hline $36 \mathrm{~m}(3 \mathrm{a})$ & $62 \mathrm{~m}$ & 148 & 146 & 123 & -25 & 117 & -31 \\
\hline $36 \mathrm{~m}(3 \mathrm{a})$ & $68 \mathrm{~m}$ & 164 & 161 & 135 & -29 & 129 & -35 \\
\hline $36 \mathrm{~m}(3 \mathrm{a})$ & $75 \mathrm{~m}$ & 180 & 177 & 149 & -31 & 142 & -38 \\
\hline $48 \mathrm{~m}(4 \mathrm{a})$ & $70 \mathrm{~m}$ & 132 & 126 & 106 & -26 & 101 & -31 \\
\hline $48 \mathrm{~m}(4 \mathrm{a})$ & $78 \mathrm{~m}$ & 148 & 141 & 118 & -30 & 113 & -35 \\
\hline $48 \mathrm{~m}(4 \mathrm{a})$ & $86 \mathrm{~m}$ & 164 & 155 & 130 & -34 & 124 & -40 \\
\hline $48 \mathrm{~m}(4 \mathrm{a})$ & $94 \mathrm{~m}$ & 180 & 169 & 142 & -38 & 135 & -45 \\
\hline $60 \mathrm{~m}(5 \mathrm{a})$ & $84 \mathrm{~m}$ & 132 & 121 & 102 & -30 & 97 & -35 \\
\hline $60 \mathrm{~m}(5 \mathrm{a})$ & $93 \mathrm{~m}$ & 148 & 135 & 114 & -34 & 108 & -40 \\
\hline $60 \mathrm{~m}(5 \mathrm{a})$ & $102 \mathrm{~m}$ & 164 & 148 & 124 & -40 & 119 & -45 \\
\hline $60 \mathrm{~m}(5 \mathrm{a})$ & $111 \mathrm{~m}$ & 180 & 162 & 136 & -44 & 130 & -50 \\
\hline $72 \mathrm{~m}(6 \mathrm{a})$ & $98 \mathrm{~m}$ & 132 & 123 & 103 & -29 & 98 & -34 \\
\hline $72 \mathrm{~m}(6 \mathrm{a})$ & $109 \mathrm{~m}$ & 148 & 137 & 115 & -33 & 110 & -38 \\
\hline $72 \mathrm{~m}(6 \mathrm{a})$ & $120 \mathrm{~m}$ & 164 & 150 & 126 & -39 & 120 & -44 \\
\hline $72 \mathrm{~m}(6 \mathrm{a})$ & $132 \mathrm{~m}$ & 180 & 164 & 138 & -42 & 131 & -49 \\
\hline $96 \mathrm{~m}(8 \mathrm{a})$ & $134 \mathrm{~m}$ & 132 & 131 & 110 & -22 & 105 & -27 \\
\hline $96 \mathrm{~m}(8 \mathrm{a})$ & $150 \mathrm{~m}$ & 148 & 148 & 124 & -24 & 118 & -30 \\
\hline $96 \mathrm{~m}(8 \mathrm{a})$ & $166 \mathrm{~m}$ & 164 & 164 & 138 & -26 & 131 & -33 \\
\hline $96 \mathrm{~m}(8 \mathrm{a})$ & $182 \mathrm{~m}$ & 180 & 180 & 151 & -29 & 144 & -36 \\
\hline
\end{tabular}

\section{Observaciones Finales}

Cuatro décadas después de su publicación, la propuesta de normas alternativas proporcionales resultaría sorprendente si la escala hubiera pasado del todo a la Historia de la Psicología. La propuesta de un modelo psicométrico general no pretende apoyar el uso actual de la escala tal y como está, salvo quizá para fines de investigación o de revisión histórica. Como se sigue empleando para la evaluación de alumnos de posible alta capacidad intelectual el problema teórico de corregir la variabilidad atípica de las normas tiene además un sentido práctico. Que la escala se siga utilizando aún erróneamente como un instrumento inalterable de medida, plantea la necesidad de explicar las causas de los grandes, progresivos y aparentemente asistemáticos desfases entre sus resultados y los del resto de las actuales escalas.

En la mayor parte de los casos la evaluación y seguimiento de la alta capacidad se inicia entre los cinco y los ocho años, precisamente en las edades con mayor distorsión de resultados y mayor desfase de las normas. Un valor de CI alterado y desfasado lleva a conclusiones y atribuciones causales incorrectas basadas en la aparente alta o extrema capacidad intelectual general del sujeto, que no se encuentra en sincronía con otras variables de su desarrollo. Las variaciones del valor del CI según el nivel inicial alcanzado y la edad de la primera evaluación, pueden llevar a atribuir la reducción del CI (reducción aparente) a diversos factores externos, como la falta de estímulos intelectuales o incluso internos, como el deterioro orgánico.

Finalmente, por el empleo de la escala Terman como prueba científica para apoyar reclamaciones judiciales de reconocimiento de posibles alumnos de alta capacidad intelectual, las normas alternativas proporcionales y los argumentos expuestos en este artículo pueden ser también útiles en el campo del Derecho.

\section{Apéndice. Determinación de Normas Alternativas Proporcionales para la Forma $\mathbf{L}$ de la Segunda Edición de la Escala Terman}

Los valores de CI se obtenían para esta edición 
considerando para todas las edades $\overline{E M}=E C$, utilizando la fórmula clásica equivalente a la constante B. Aquí se consideran rendimientos medios las medias pulidas del CI que figuran en el "Cuadro V" del manual (Terman, L. M. \& Merrill, M. A. 1943, p. 39). La dispersión $S_{E M}$ se calcula a partir de los datos de la columna variabilidad del CI que aparece en el Cuadro VII (p.45), utilizando la siguiente proporción:

$\overline{\frac{E M}{S_{E M}}}-\frac{100}{S_{C I}}$
Se aplica el mismo procedimiento para la normalización explicado en el artículo para la Tercera Edición, con los resultados indicados en la Tabla A1. La constante de las normas alternativas proporcionales resulta sistemáticamente menor que la constante ideal y los rendimientos medios quedan por tanto incrementados, especialmente en las edades en torno a los 6 años. Estos efectos son significativos para las EC menores a los ocho años, alcanzando su máximo en torno a los seis años, como ocurrirá posteriormente en las normas de la Tercera Edición.

Tabla A1. Escala Terman. Normas Alternativas Proporcionales a las de la Segunda Edición Forma L, entre las EC de 3 y 14 años $\left(S_{C I}{ }_{C l}=16.66\right)$

\begin{tabular}{|c|c|c|c|c|c|c|c|c|c|c|c|}
\hline $\mathrm{EC}$ & $B$ & $\overline{E M}$ & $S_{E M}$ & $a$ & $b$ & $E M_{X}$ & $C I_{X}$ & $B^{\prime}$ & $b^{\prime}$ & $\overline{E M}$, & $S_{E M^{\prime}}$ \\
\hline 3 años & 2.78 & 38.8 & 6.84 & 5.44 & 2.44 & 41.04 & 105.44 & 2.57 & 1.05 & 38.9 & 6.49 \\
\hline 3 a $6 \mathrm{~m}$ & 2.38 & 44.1 & 7.27 & -1.25 & 2.30 & 43.60 & 98.75 & 2.26 & 0.99 & 44.1 & 7.36 \\
\hline 4 años & 2.08 & 50.3 & 8.11 & -3.25 & 2.05 & 48.67 & 96.75 & 1.99 & 0.97 & 50.3 & 8.38 \\
\hline 4 a $6 \mathrm{~m}$ & 1.85 & 55.9 & 8.75 & -6.48 & 1.90 & 52.49 & 93.52 & 1.78 & 0.93 & 56.1 & 9.35 \\
\hline 5 años & 1.67 & 61.9 & 8.52 & -21.00 & 1.96 & 51.12 & 78.99 & 1.54 & 0.79 & 64.7 & 10.79 \\
\hline 5 a $6 \mathrm{~m}$ & 1.51 & 67.4 & 9.44 & -19.11 & 1.77 & 56.63 & 80.89 & 1.43 & 0.81 & 70.0 & 11.67 \\
\hline 6 años & 1.39 & 73.0 & 9.00 & -35.20 & 1.85 & 54.00 & 64.80 & 1.20 & 0.65 & 83.3 & 13.89 \\
\hline 7 años & 1.19 & 85.4 & 13.61 & -4.62 & 1.22 & 81.65 & 95.37 & 1.17 & 0.95 & 85.6 & 14.27 \\
\hline 8 años & 1.04 & 98.7 & 15.17 & -8.43 & 1.10 & 91.01 & 91.56 & 1.01 & 0.92 & 99.4 & 16.57 \\
\hline 9 años & 0.93 & 112.0 & 17.71 & -5.38 & 0.94 & 106.27 & 94.61 & 0.89 & 0.95 & 112.3 & 18.72 \\
\hline 10 años & 0.83 & 125.3 & 19.80 & -5.45 & 0.84 & 118.80 & 94.55 & 0.80 & 0.94 & 125.6 & 20.94 \\
\hline 11 años & 0.76 & 137.4 & 23.76 & 3.61 & 0.70 & 142.56 & 103.61 & 0.73 & 1.04 & 137.6 & 22.93 \\
\hline 12 años & 0.69 & 149.8 & 28.80 & 13.34 & 0.58 & 172.80 & 113.33 & 0.66 & 1.13 & 152.5 & 25.41 \\
\hline 13 años & 0.64 & 160.4 & 27.92 & 4.29 & 0.60 & 167.54 & 104.28 & 0.62 & 1.04 & 160.7 & 26.78 \\
\hline 14 años & 0.59 & 172.4 & 27.05 & -6.21 & 0.62 & 162.29 & 93.79 & 0.58 & 0.94 & 173.0 & 28.84 \\
\hline
\end{tabular}

\section{Extended Summary}

In this article I argue the thesis that the Terman scale tends to generate distributions of typical derivative markings for the different chronological age levels (CA), if the passing criteria are sufficiently adjusted and the samples are representative enough. This type of scales have a common origin at $M A_{0}=0$ (MA: mental age) and the relationship $\overline{M A} / S_{M A}=6$ or every $C A$.

The dispersion which the performance (MA) and the intelligence quotient values (IQ) tend to are inferred from the scale structure, which I qualify as natural dispersion. Its resolution allows us to generalize the psychometric model of the Terman scale and to determine proportional alternative rules, which eliminate the atypical variability of the IQ values of the Second and of the two versions of the Third Edition.

\section{IQ Estimation}

\section{Natural Dispersion of Performance and its Consequences}

The terminology that sets up execution $I Q$ against deviation $I Q$ frequently produces confusion in thinking, specifically that the Terman scale measures IQ values and their dispersion $\left(S_{I Q}\right)$ directly, when in reality they gather performance (MA) and the dispersion of this performance $\left(S_{M A}\right)$. The source of atypi- 
cal variability is found in the performance, where it must be corrected to avoid transferring it to the IQ.

The scale can be considered as the union of six longitudinal and parallel series, each one having a test per IQ level. On this type of distributions the mean coincides with the standard deviation. Each individual has a potentially different performance on each series, with its performance being on the total scale $\left(M A_{i}\right)$ the addition of partial performance on the six series. Since on the ideal adjustment the six tests of each IQ level are equivalent, the average performance of each series $\left(\overline{m a}_{p}\right)$ is equal, and therefore its standard deviations $\left(S_{m a_{p}}\right)$ are also equal. In conclusion, for each IQ level of the ideally adjusted scale, the average performance is six times larger than the standard deviation:

The natural dispersion to which the scale (called $S$ ' from now on) tends depends ideally on its structure, that is, on the number of tests per IQ level ( $p)$, not on the population or times over which rules are determined. In identifying the IQ scale with the one of the ideal group of $\overline{M A}=100$, the IQ natural dispersion on the Terman scale is $S_{I Q}{ }_{I Q}-16.66$. In other words, the coefficient of variation (CV) for all age levels is $1 / 6$. Determining the natural dispersion of the performance makes the proportional adjustment of the performance and the determining the IQ without distortions of the rules possible.

\section{General Psychometric Model of the Terman Scale}

The substitution on the classical equation of CA (measurement in months of time) by $\overline{M A}$ (measurement in moths of performance) and the determination of the natural dispersion of the performance allows to generalize the psychometric model. The IQ is now identified with the performance of a group of ideal age with $\overline{M A}=100$.

The general psychometric model proposed here is determined by the following assumptions for all CA levels:

1. normal distributions of performance $(M A)$.

2. $\overline{M A} / S_{M A}=6$.
If the dispersions are adjusted to their ideal values, the IQ is equally obtained from the relationship $S_{I Q} / S_{M A}$. The constant $B=100 / C A$ of the ideal model is substituted by the constant $B^{\prime}=100 / M A$, maintaining the ratio between performance and $I Q$ values.

\section{Atypical Variability of the Third Edition Rules}

The Third Edition of the Terman Scale appeared in 1960, with an single combined form L-M. A decade later (1972), R. L. Thorndike worked on the latest rules. Essentially, the common IQ distribution was obtained, with $\overline{I Q}=100$ and $S_{I Q}=16$, using the transformation equations of typical markings. The rules show deviation at different levels for each CA, which increase as they separate from the average.

\section{Determination of Proportional Alternative Rules}

To make the general model operative, the constant $B$ ' must be determined for each CA level. The 1972 rules are modified with $S_{I Q}{ }_{I Q}-16.66$. The new constants $a$ and $b$ are indicated on Table 1 .

\section{Calculation of constant $B$}

We take the performance $M A_{X}$ as reference for the proportional correction of rules for each $\mathrm{CA}$, which corresponds to the IQ of 100 before adjusting $a$, that is $M A_{X}=6 \times S_{M A}$. This performance $M A_{X}$, instead of $\overline{M A}$, makes constant $a$ null. The corresponding $I Q_{X}$ is likewise obtained. The relationship between $I Q_{X}$ and $M A_{X}$ determines the sought constant $B$ ', which allows the calculation of the IQ without distortion $\left(I Q^{\prime}\right)$ :

$B^{\prime}=\frac{I Q_{X}}{M A_{X}}$

$I Q_{i}^{\prime}=B^{\prime} \times M A_{i}$

The obtained alternative rules, adjusting the sca- 
les from $M A_{X}$ are strictly proportional and, rounding the calculated values, $\overline{M A}$ are not modified in many cases or they hardly vary. However, constant $B^{\prime}$ is smaller than constant $b$, for different CA levels, especially at younger ages. The values $M A_{X}, I Q_{X}$, constants $B$ 'y $b$ ' (defined on the next section), and the corrected parameters $\left(\overline{M A}\right.$, and $\left.S_{M A}^{\prime}\right)$ are indicated on Table 2.

\section{General Formula of the Execution IQ'}

The obtained constant $B$ ' directly transforms performance $M A_{i}$ in proportional $I Q_{X}$ values. It is written as a function of $\overline{M A}$ and $S_{M A}$ as follows:

$I Q_{i}^{\prime}=\left[2-\frac{\overline{M A}}{6 \times S_{M A}}\right] \times\left[\frac{S_{I Q}^{\prime}}{S_{M A}}\right] \times M A_{i}$

Labeling $b$ ' and $b$ the two multiplying factors in respectively:

$I Q_{i}^{\prime}=b^{\prime} \times b \times M A_{i}$

The different adjustment procedures of the rules are shown on Graph 2. According to the second condition of the General Psychometric Model, with the increase in the dispersion of performance (slope $B^{\prime}<$ slope $\left.b\right)$ the average performance $(\overline{M A},>\overline{M A})$ increases. Constant $b$ ' proportionally increases the median of the original distribution (which is $M A_{X}$ ) and it turns into the median and average performance of the corrected scale $(\overline{M A})$. Therefore, the new $\overline{M A}$ ' does not come from, but from the performance, reference to determine the alternative rules.

\section{IQ Dynamics}

It is important to point out that individuals do not directly increase their IQ, but rather their performance on the scales used to measure it (in MA units in this case). If the rules become outdated, the obtained IQ values come out larger that they should be. On the other hand, the general improvement of the pop- ulation in the execution of the intelligence scales is not due to the uniform progress at all levels of performance. In graphical terms, the normal curve of performance (and therefore IQ) does not translate itself towards its left in a block translation. The psychometric model of the Terman scale associates the general increase of performance $(\overline{M A})$, which is normally distributed, with a proportional increase of its dispersion $\left(S_{M A}\right)$. Graphically, the normal curve representing the IQ stays fixed at the minimal execution levels and it translates itself towards the left by expansion increasing simultaneously the average and the dispersion of its values.

The disparity of the rules from 1960 to 1972 can bring relevant information to the debate about how much IQ increases as time moves on. Table 3 shows the corrected average performance $(\overline{M A}$ ') for 1960 and 1972 rules. The increase of the average performance in a decade, for ages between three and fourteen, was globally close to five per cent. We can observe that the increase is significantly larger for younger ages. The more stable IQ values are shown to be the ones corresponding to the lower performance (for just those cases in which the scale is no longer used). Finally, the values that suffer athe largest change are the highest for the youngest individuals (in just those cases in which the scale is still used). Table 4 indicates some examples of IQ correction and later update. The offered calculations should be understood as general tendencies, without giving them an accuracy they do not have.

\section{Final Comments}

The suggestion of a general psychometric model does not try to support the use of the scale such as it is, except perhaps for research purposes or history review. To continue with the incorrect use of the scale as an unchangeable measurement tool, creates the need to explain the causes of the large, progressive, and apparently asymmetrical discrepancies between its results and those of the rest of the present scales. 
In most cases, the evaluation and monitoring of high capacity starts between five and eight years old, at just the ages with the largest distortion in the results and the largest discrepancies in the rules. An altered and outdated IQ value leads to incorrect causal conclusions and attributions. The variations of the IQ value according to the reached initial level and the age of the first evaluation can also wrongly lead to assign the reduction in IQ (apparent reduction) to different external or internal factors.

Finally, the use of the Terman scale as scientific proof to support legal claims in recognizing potential students with high intellectual capability, makes the arguments presented in this article also useful in Law.

The Appendix at the end shows the data of the adjustment of the factor rules and the proposed proportional correction for the Second Edition of the scale.

\section{Referencias}

Angoff W. H. (1971). Scales, Norms and Equivalent Scores, en W. L. Thorndike Ed., Educational

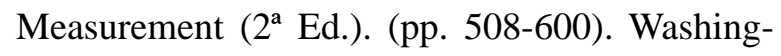
ton, D.C.: American Council of Education.

McNemar, Q. (1942). The revisión of the StanfordBinet Scale. An Analysis of Standardization Data. Houghton Mifflin Company. Boston.

Terman, L. M. y Merrill, M. A. (1943). Medida de la Inteligencia. Método para el empleo de las pruebas del Stanford-Binet nuevamente revisadas. Traducción y adaptación de Germain, J. EspasaCalpe S.A. Madrid.

Terman, L. M. y Merrill, M. A. (1975). Medida de la Inteligencia. Método para el empleo de las pruebas del Stanford-Binet. Tercera revisión Formas L y $\mathrm{M}$ reunidas. Traducción y adaptación de Germain, J. Espasa-Calpe S.A. Madrid.

Manuscrito recibido: 14/04/2011

Revisión recibida: 18/05/2011

Manuscrito aceptado: 24/05/2011 